\title{
Herpes Simplex Virus Encephalitis in a Healthy Lady
}

\section{Abstract}

The case describes a middle aged lady with herpes simplex encephalitis. The highlight of the case is the MRI which depicts all the typical features of herpes encephalitis. Herpes virus is the most common cause of encephalitis worldwide. It is very important to recognize this typical MRI picture and initiate Acyclovir therapy at the earliest because, early initiation of therapy is the single factor which affects the prognosis of this serious neurological illness.

Keywords: Herpes simplex encephalitis; Viral encephalitis; Magnetic resonance imaging; Herpes simplex virus

Received: May 28, 2018; Accepted: July 03, 2018; Published: July 10, 2018

\section{Case Report}

A 49-year-old female presented with high grade fever of 2-days duration followed by altered sensorium for the past 8-days and one episode of generalized seizure. On arrival at the emergency department she had a respiratory arrest and was intubated. On examination she was drowsy but arousable. She was afebrile and hemodynamically stable. Pupils were equal and reactive. Blood investigations and serum electrolytes were normal. Cerebrospinal fluid (CSF) study showed 5 cells, 100\% lymphocytes, protein of $20 \mathrm{mg} / \mathrm{dl}$ and sugar of $106 \mathrm{mg} / \mathrm{dl}$. Electroencephalography (EEG) showed diffuse slowing of background activity. Magnetic resonance imaging (MRI) of the brain showed $\mathrm{T} 2$ and fluid-attenuated inversion recovery (FLAIR) hyperintensities in bilateral insular cortex, cingulate gyrus (Figure 1A), medial temporal lobes (Figure 1B) and orbitofrontal gyrus of both frontal lobes (Figure 1C) with diffusion restriction (Figure $\mathbf{2 A}$ and $\mathbf{2 B}$ ), typical of herpes simplex encephalitis (HSE). CSF polymerase chain reaction (PCR) for herpes simplex was positive. She was treated with intravenous acyclovir at a dose of $500 \mathrm{mg}$ eight hourly with dexamethasone $4 \mathrm{mg}$ eight hourly for 21 days, but she succumbed to the illness after 1-month.

\section{Discussion}

Fever, altered sensorium and seizures should raise the possibility of encephalitis. Herpes simplex virus-1 (HSV-1) is the most common cause of sporadic encephalitis. It is seen in all age groups with one-third of cases occurring in children and adolescents. HSV-1 enters the brain through olfactory bulb and primarily infects the areas where olfactory pathway relays [1]. Various host factors are recognized which predispose to HSE. Toll

\section{Joe James ${ }^{1 *}$, Jinu Johnson ${ }^{2}$, James Jose ${ }^{1}$ and Thulaseedharan NK $^{2}$}

\author{
${ }^{1}$ Department of Neurology, Government \\ Medical College Kozhikode, Kerala, India \\ ${ }^{2}$ Department of Internal Medicine, \\ Government Medical College Kozhikode, \\ Kerala, India
}

\section{*Corresponding author: Joe James}

Department of Neurology, Government Medical College Kozhikode, Kerala, India.

§ drjoejames@gmail.com

Tel: +918281312815

\author{
Citation: James J, Johnson J, Jose J, \\ Thulaseedharan NK (2018) Herpes Simplex \\ Virus Encephalitis in a Healthy Lady. J Rare \\ Disord Diagn Ther. Vol.4 No.3:15
}

like receptor-3 (TLR-3) is expressed in the CNS and synthesize interferon, which prevent spread of infection from olfactory epithelium to CNS. Defects in TLR-3 pathway can predispose to HSE. Herpes virus typically involves the orbitofrontal lobes, medial frontal lobe, insular cortex and medial temporal lobes [2]. Involvement of basal ganglia or substantia nigra, which is typical of arboviral encephalitis, excludes HSE. Fever, altered sensorium, focal neurological deficits like aphasia, hemiparesis, seizures are the common presentation of HSE. Behavioral and psychiatric symptoms may precede the onset of other neurological signs in HSE. These include hypomania due to involvement of limbic system, hypersexuality, hyperorality and visual agnosia (KlüverBucy syndrome). Other described symptoms include urinary incontinence and rarely skin rash. CSF usually shows lymphocytic pleocytosis but a normal CSF does not exclude the diagnosis and simply implies that there is no meningeal inflammation [3]. The most sensitive method for diagnosing HSE is CSF PCR for viral DNA. It has a sensitivity of $98 \%$ and specificity of $94 \%$ to $100 \%$ [4]. Antibodies to HSV glycoprotein B can be detected in CSF after 10 to 14 days and has a sensitivity of $97 \%$ and specificity of $100 \%$ but is not widely available [5]. HSV antigen can also be 

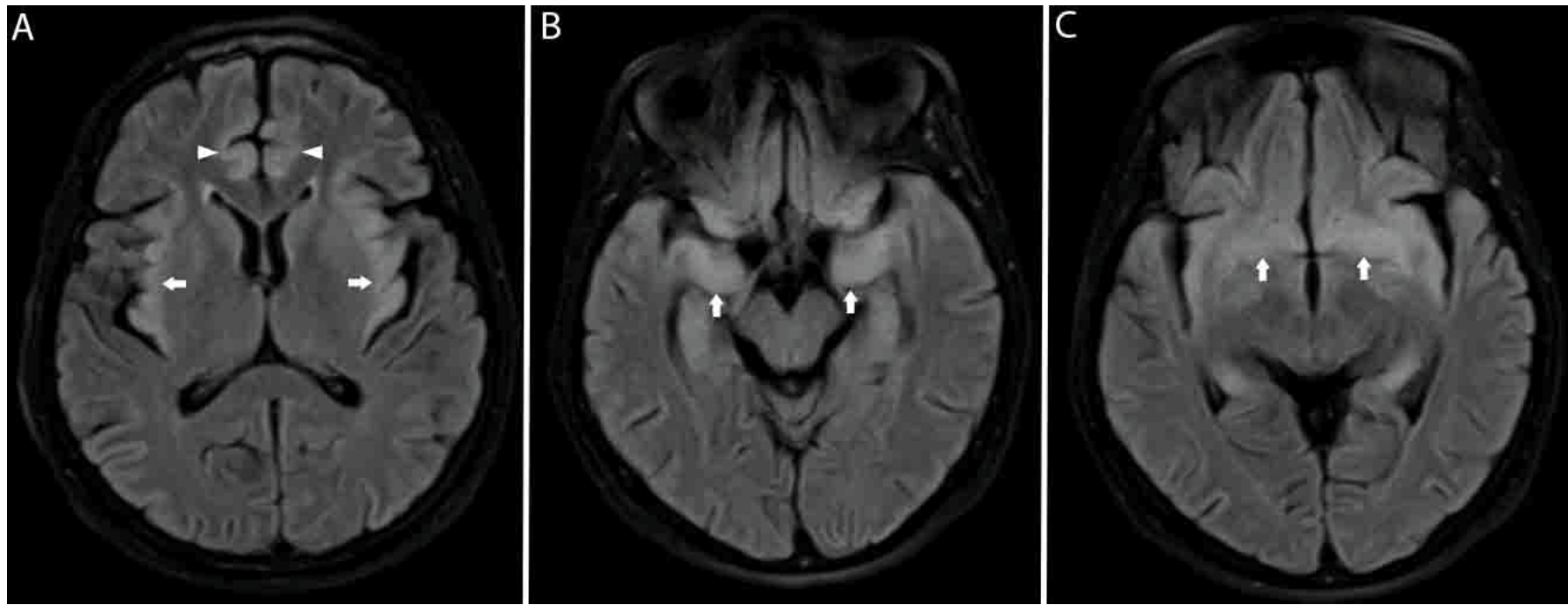

Figure 1 (A) Axial Magnetic Resonance Imaging showing T2-FLAIR hyperintensity in bilateral inuslar cortex (arrows) and cingulate gyrus (arrowhead), (B) bilateral medial temporal lobes (arrows) and (C) orbitofrontal gyrus of both frontal lobes (arrows).
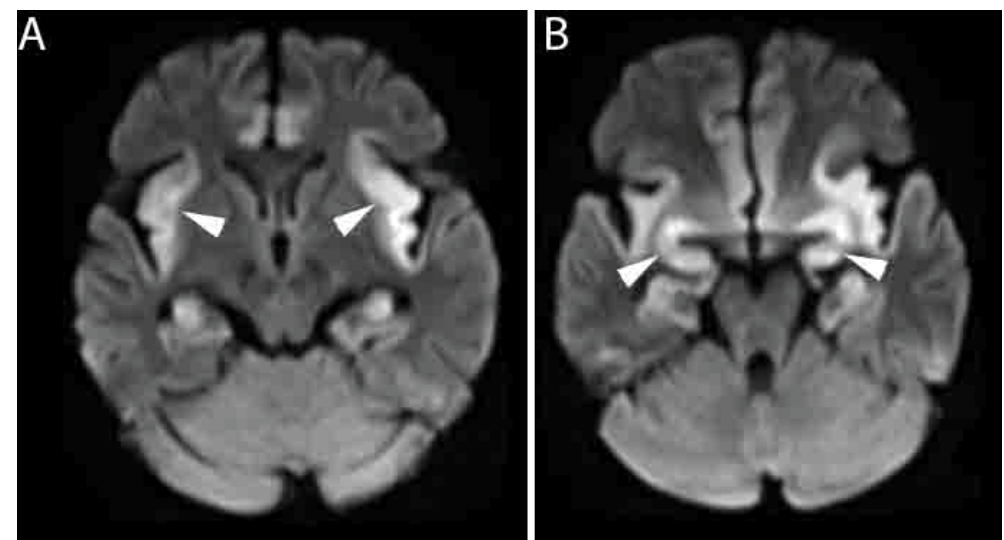

Figure 2 (A) MRI Diffusion Weighted Image showing diffusion restriction in both insular cortex (arrowheads) and (B) both medial tempora lobes (arrowheads).

detected in the CSF, but sensitivity and specificity of this test is lower than PCR. EEG is abnormal in upto $80 \%$ cases and may show periodic lateralised epileptiform discharges. However, this is not very specific and can occur in many other neurological illnesses. With a normal CSF and non-specific EEG, an MRI finding of hyperintensity in inferior frontal and medial temporal lobe is diagnostic of herpes simplex encephalitis. Newer MRI techniques like diffusion tensor imaging (DTI) can provide additional directional diffusion data over a normal diffusion weighted sequence [6]. In a study of six PCR positive cases, DTI analysis showed reduced mean diffusivity (MD) and increased fractional anisotropy (FI) values in early phase. After 2-weeks, the $\mathrm{MD}$ increases and $\mathrm{FI}$ decreases, consistent with vasogenic edema. However, larger studies are needed before this can be used in routine clinical practice. Differential diagnosis in this setting include other viral encephalitis (West Nile virus, Japanese encephalitis, Enteroviruses among others), autoimmune and paraneoplastic encephalitis and acute disseminated encephalomyelitis, especially in children. Early initiation of acyclovir is the most important factor affecting prognosis of this serious illness [7]. Early treatment is defined as initiation of acyclovir before loss of consciousness or within 24 hours of onset of symptoms. It is recommended to give acyclovir for 14 to 21 days in confirmed cases of HSE, as a shorter course has been associated with relapses [8]. One study evaluated the efficacy of Valacyclovir at a dose of $1 \mathrm{~g}$ orally thrice daily for 21 days, in four patients with HSE [9]. The pharmacokinetic study showed that therapeutic concentrations of valacyclovir were achieved and maintained throughout the duration of therapy, although the CSF concentrations dropped over time, likely due to resolution of meningeal inflammation and reduction in blood-brain barrier permeability. But, larger studies are required before valacyclovir can be recommended for HSE. Alternate agents like Ganciclovir and Foscarnet have demonstrated in-vitro activity against HSV, but studies are lacking to give any recommendations. Even with appropriate therapy mortality rate is a high as $30 \%$ [1]. Long term sequelae of HSE include neuropsychiatric and cognitive defects, including anterograde amnesia, dysnomia, Klüver-Bucy syndrome 
and epilepsy [8]. HSE may expose sequestrated neuro-antigens, with consequent development of antibodies and autoimmune encephalitis. Upto $30 \%$ of patients with HSE have anti N-methyl-
D-aspartate receptor (NMDAR) antibodies and a proportion of the survivors develop anti-NMDAR encephalitis and hence long term follow up of these patients is necessary [10].

\section{References}

1 Levitz RE (1998) Herpes simplex encephalitis: a review. Heart Lung 27: $209-212$.

2 Kennedy PGE, Chaudhuri A (2002) Herpes simplex encephalitis. J Neurol Neurosurg Psychiatry 73: 237-238.

3 Rawal G, Yadav S, Wani UR, Ambastha AK (2015) HSV Encephalitis with Normal CSF - A Case Report with Review of Literature. J Clin Diagn Res 9: OD06-OD07.

4 Boivin G (2004) Diagnosis of herpes virus infections of the central nervous system. Herpes 2: 48A-56A.

5 Kahlon J, Chatterjee S, Lakeman FD, Lee F, Nahmias AJ, et al. (1987) Detection of antibodies to herpes simplex virus in the cerebrospinal fluid of patients with herpes simplex encephalitis. J Infect Dis 155: 38-44.

6 Herweh C, Jayachandra M, Hartmann M, Gass A, Sellner J, et al. Quantitative diffusion tensor imaging in herpes simplex virus encephalitis. J Neurovirol 13: 426-432.

7 McGrath N, Anderson NE, Croxson MC, Powell KF (1997) Herpes simplex encephalitis treated with acyclovir: diagnosis and long term outcome. J Neurol Neurosurg Psychiatry 63: 321-326.

8 Gordon B, Selnes OA, Hart J, Hanley DF, Whitley RJ, et al. (1990) Long-term cognitive sequelae of acyclovir-treated herpes simplex encephalitis. Arch Neurol 47: 646-647.

9 Pouplin T, Pouplin JN, Van Toi P, Lindegardh N, Hien TT, et al. (2011) Valacyclovir for herpes simplex encephalitis. Antimicrob Agents Chemother 55: 3624-3626.

10 Morris NA, Kaplan TB, Linnoila J, Cho T (2016) HSV encephalitisinduced anti-NMDAR encephalitis in a 67-year-old woman: report of a case and review of the literature. J Neurovirol 22: 33-37. 